\title{
AMENDMENTS
}

\section{Author Correction: Optogenetic control of cofilin and $\alpha$ TAT in living cells using Z-lock}

Orrin J. Stone, Neha Pankow, Bei Liu, Ved P. Sharma, Robert J. Eddy, Hui Wang, Andrew T. Putz, Frank D. Teets, Brian Kuhlman (D, John S. Condeelis and Klaus M. Hahn (D)

Correction to: Nature Chemical Biology https://doi.org/10.1038/s41589-019-0405-4, published online 18 November 2019

In the version of this article originally published, several images were incorrectly set. In Supplementary Fig. 1d, the gels for cofilin and actin were switched. The associated full gels, correct in the original submission, are in Supplementary Fig. 3. In Fig. 4b, the bands for " $\alpha$ TAT core" and for "DN $\alpha$ TAT" were stretched when copied from the original gel to the figure. The original and corrected versions of Fig. $4 \mathrm{~b}$ are shown below. The errors have been corrected in the HTML and PDF versions of the paper and in the Supplementary Information file.

Corrected

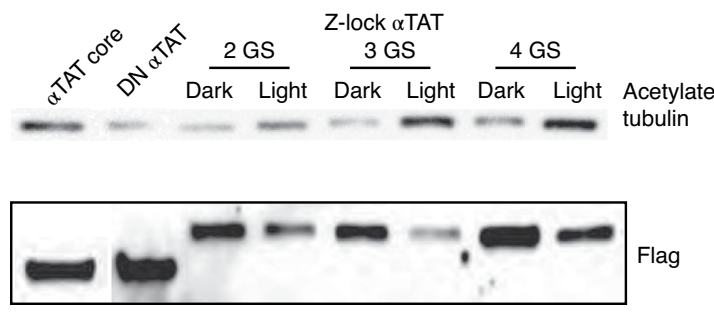

Original

b

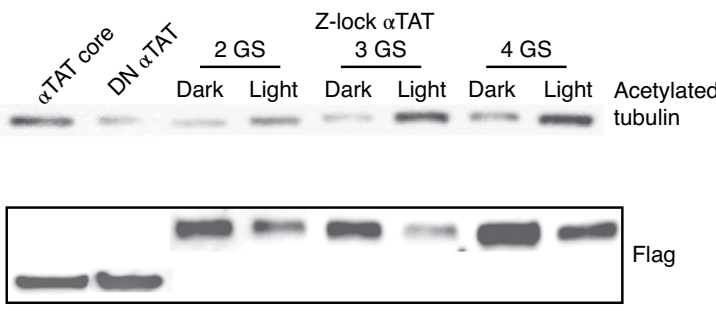

Fig. 4 | Original and Corrected.

Published online: 21 July 2020

https://doi.org/10.1038/s41589-020-0614-x

(c) The Author(s), under exclusive licence to Springer Nature America, Inc. 2020

\section{Author Correction: The bottromycin epimerase BotH defines a group of atypical $\boldsymbol{\alpha} / \boldsymbol{\beta}$-hydrolase-fold enzymes}

Asfandyar Sikandar, Laura Franz (1), Sebastian Adam, Javier Santos-Aberturas @i , Liliya Horbal, Andriy Luzhetskyy, Andrew W. Truman (1), Olga V. Kalinina and Jesko Koehnke (D)

Correction to: Nature Chemical Biology https://doi.org/10.1038/s41589-020-0569-y, published online 29 June 2020

In the version of this article originally published, some information regarding Fig. 5 was switched around. The figure citation in the sentence "Based on the biochemical and structural data, we propose the following mechanism for epimerization in bottromycin biosynthesis (Fig. 5b)" should refer to Fig. 5 a instead of 5b. Additionally, the text for panels a and $\mathbf{b}$ in the legend for Fig. 5 were swapped. The legend should read "a, Proposed mechanism for the epimerization of $\mathbf{3 b}$ to yield $\mathbf{3 a}$. BotH residues are labeled in black and substrate residues in blue. Hydrogen bonds are shown as dashed lines. Ordered water molecules surrounding the Asp7 carboxy group are not shown for clarity (see Extended Data Fig. 5 for details). b, Proposed role of BotH in self-resistance. A rise of intracellular bottromycin concentrations leads to inhibition of BotH epimerase activity, which may in turn prevent self-poisoning of the producing strain. BotH may also act as an intracellular buffer to store bottromycins." The errors have been corrected.

Published online: 15 July 2020

https://doi.org/10.1038/s41589-020-0624-8

(c) The Author(s), under exclusive licence to Springer Nature America, Inc. 2020 